Article

\title{
An Eigenvalue Inclusion Set for Matrices with a Constant Main Diagonal Entry
}

\author{
Weiqian Zhang ${ }^{1}$ and Chaoqian $\mathrm{Li}^{2, *}$ \\ 1 School of Mathematics and Statistics, Zhoukou Normal University, Zhoukou 466399, China; \\ weiqianxiaozhu@163.com \\ 2 School of Mathematics and Statistics, Yunnan University, Kunming 650091, China \\ * Correspondence: lichaoqian@ynu.edu.cn
}

Received: 27 October 2018; Accepted: 11 December 2018; Published: 12 December 2018

\begin{abstract}
A set to locate all eigenvalues for matrices with a constant main diagonal entry is given, and it is proved that this set is tighter than the well-known Geršgorin set, the Brauer set and the set proposed in (Linear and Multilinear Algebra, 60:189-199, 2012). Furthermore, by applying this result to Toeplitz matrices as a subclass of matrices with a constant main diagonal, we obtain a set including all eigenvalues of Toeplitz matrices.
\end{abstract}

Keywords: eigenvalue; matrices with a constant main diagonal; Toeplitz; inclusion set

\section{Introduction}

Eigenvalue localization is an important topic in Matrix theory and its applications. Many eigenvalue inclusion sets for a matrix $A=\left[a_{i j}\right] \in \mathbb{C}^{n \times n}[1-11]$ have been established, such as the well-known Geršgorin set [5,11] and the Brauer set [1,11]. However, as Melman [9] pointed out, for the special class of matrices with a constant main diagonal (c.m.d.), both the Geršgorin and Brauer sets each consists of a single disc, a rather uninteresting outcome. In fact, if a matrix $A=\left[a_{i j}\right] \in \mathbb{C}^{n \times n}$ satisfies $a_{11}=a_{22}=\cdots=a_{n n}=\bar{a}$, then both $\Gamma(A)$ and $\mathcal{K}(A)$ reduce, respectively, to the following forms:

$$
\Gamma(A)=\left\{z \in \mathbb{C}:|z-\bar{a}| \leq \max _{i \in N} r_{i}(A)\right\},
$$

and

$$
\mathcal{K}(A)=\left\{z \in \mathbb{C}:|z-\bar{a}| \leq \max _{i, j \in N, i \neq j} \sqrt{r_{i}(A) r_{j}(A)}\right\},
$$

where $r_{i}(A)=\sum_{j \neq i}\left|a_{i j}\right|$ and $N=\{1,2, \ldots, n\}$. Obviously, the Geršgorin and Brauer sets are just discs [9].

To localize all eigenvalues of matrices with a c.m.d. more precisely, Melman also [9] gave an eigenvalue inclusion set (see Theorem 1), which is tighter than $\Gamma(A)$ and $\mathcal{K}(A)$.

Theorem 1 ([9] Theorem 2.1). Let $A=\left[a_{i j}\right] \in \mathbb{C}^{n \times n}$ with $a_{i i}=\bar{a}$ for all $i \in N, n \geq 2$. Let $\sigma(A)$ be the spectrum of the matrix $A$, that is, $\sigma(A)=\{\lambda \in \mathbb{C}: \operatorname{det}(\lambda I-A)=0\}$. Then,

$$
\sigma(A) \subseteq \Omega(A)=\bigcup_{i \in N} \Omega_{i}(A)
$$

where $A_{0}=A-\bar{a} I,\left(A_{0}^{2}\right)_{i j}$ denotes the $(i, j)$ th entry of $A_{0}^{2}$ and

$$
\Omega_{i}(A)=\left\{z \in \mathbb{C}:\left|z-\bar{a}-\sqrt{\left(A_{0}^{2}\right)_{i i}}\right|\left|z-\bar{a}+\sqrt{\left(A_{0}^{2}\right)_{i i}}\right| \leq r_{i}\left(A_{0}^{2}\right)\right\} .
$$


Furthermore, $\Omega(A) \subseteq \mathcal{K}(A) \subseteq \Gamma(A)$.

In [7], Li and Li provided two tighter sets including all eigenvalues of a matrix with a c.m.d. (see Theorems 2 and 3).

Theorem 2 ([7] Theorem 2.4). Let $A=\left[a_{i j}\right] \in \mathbb{C}^{n \times n}$ with $a_{i i}=\bar{a}$ for all $i \in N, n \geq 2$. Then,

$$
\sigma(A) \subseteq \Omega^{1}(A)=\bigcap_{0 \leq \alpha \leq 1} \bigcup_{i \in N} \Omega_{i}^{1 \alpha}(A),
$$

where

$$
\Omega_{i}^{1 \alpha}(A)=\left\{z \in C:\left|z-\bar{a}-\sqrt{\left(A_{0}^{2}\right)_{i i}}\right|\left|z-\bar{a}+\sqrt{\left(A_{0}^{2}\right)_{i i}}\right| \leq \alpha r_{i}\left(A_{0}^{2}\right)+(1-\alpha) c_{i}\left(A_{0}^{2}\right)\right\} .
$$

Theorem 3 ([7] Theorems 2.5 and 2.7). Let $A=\left[a_{i j}\right] \in \mathbb{C}^{n \times n}$ with $a_{i i}=\bar{a}$ for all $i \in N, n \geq 2$. Then,

$$
\sigma(A) \subseteq \Omega^{2}(A)=\bigcap_{0 \leq \alpha \leq 1} \bigcup_{i \in N} \Omega_{i}^{2 \alpha}(A),
$$

where

$$
\Omega_{i}^{2 \alpha}(A)=\left\{z \in C:\left|z-\bar{a}-\sqrt{\left(A_{0}^{2}\right)_{i i}}\right|\left|z-\bar{a}+\sqrt{\left(A_{0}^{2}\right)_{i i}}\right| \leq\left(r_{i}\left(A_{0}^{2}\right)\right)^{\alpha}\left(c_{i}\left(A_{0}^{2}\right)\right)^{1-\alpha}\right\} .
$$

Furthermore,

$$
\Omega^{2}(A) \subseteq \Omega^{1}(A) \subseteq\left(\Omega(A) \bigcap \Omega\left(A^{T}\right)\right) \subseteq\left(\mathcal{K}(A) \bigcap \mathcal{K}\left(A^{T}\right)\right) \subseteq\left(\Gamma(A) \bigcap \Gamma\left(A^{T}\right)\right) .
$$

In this paper, we first give a sufficient condition for non-singular matrices, which leads to a new set including all eigenvalues of matrices with a c.m.d. As an application, in Section 3, we apply the result obtained in Section 2 to Toeplitz matrices as a subclass of matrices with a c.m.d. and obtain a new eigenvalue inclusion set. All the new eigenvalue inclusion sets are proved to be tighter than those in [9].

\section{A New Eigenvalue Inclusion Set for Matrices with a c.m.d.}

In this section, we present a new eigenvalue inclusion set for matrices with a c.m.d. First, a sufficient condition for non-singular matrices is given.

Lemma 1. For any $A=\left[a_{i j}\right] \in \mathbb{C}^{n \times n}$ with $a_{i i}=\bar{a}$ for all $i \in N$, and $n \geq 2$, if

$$
\left|\bar{a}^{2}-\left(A_{0}^{2}\right)_{i i}\right|\left|\bar{a}^{2}-\left(A_{0}^{2}\right)_{j j}\right|>r_{i}\left(A_{0}^{2}\right) r_{j}\left(A_{0}^{2}\right),
$$

where $A_{0}=A-\bar{a} I$, then $A$ is non-singular.

Proof. Suppose on the contrary that $A=\left[a_{i j}\right] \in \mathbb{C}^{n \times n}$ satisfies Inequality (1) and is singular, then there is an $x=\left[x_{1}, x_{2}, \ldots, x_{n}\right]^{T} \in \mathbb{C}^{n}$, with $x \neq 0$, such that $A x=0$. Let

$$
0<\left|x_{t}\right| \geq\left|x_{s}\right| \geq \max \left\{\left|x_{k}\right|: k \in N, k \neq s, k \neq t\right\} .
$$

Note that $A_{0}=A-\bar{a} I$. Then, $A_{0} x=-\bar{a} x$, which leads to $A_{0}^{2} x=\bar{a}^{2} x$, equivalently, $\left(A_{0}^{2}-\bar{a}^{2} I\right) x=0$. This implies that for all $i \in N$,

$$
\left(\left(A_{0}^{2}\right)_{i i}-\bar{a}^{2}\right) x_{i}=-\sum_{j \in N, j \neq i}\left(A_{0}^{2}\right)_{i j} x_{j}
$$


Hence,

$$
\left|\left(A_{0}^{2}\right)_{i i}-\bar{a}^{2}\right|\left|x_{i}\right| \leq \sum_{j \in N, j \neq i}\left|\left(A_{0}^{2}\right)_{i j}\right|\left|x_{j}\right|, \forall i \in N .
$$

Taking $i=t$, Inequality (2) becomes

$$
\left|\left(A_{0}^{2}\right)_{t t}-\bar{a}^{2}\right|\left|x_{t}\right| \leq \sum_{j \in N, j \neq t}\left|\left(A_{0}^{2}\right)_{t j}\right|\left|x_{j}\right| \leq r_{t}\left(A_{0}^{2}\right)\left|x_{s}\right| .
$$

If $\left|x_{s}\right|=0$, then Inequality (3) reduces to $\left|\left(A_{0}^{2}\right)_{t t}-\bar{a}^{2}\right|\left|x_{t}\right|=0$, implying that $\left|\left(A_{0}^{2}\right)_{t t}-\bar{a}^{2}\right|=0$. However, this contradicts Inequality (1). Hence, $\left|x_{s}\right|>0$. We now take $i=s$ in Inequality (3), and obtain similarly

$$
\left|\left(A_{0}^{2}\right)_{s s}-\bar{a}^{2}\right|\left|x_{s}\right| \leq r_{s}\left(A_{0}^{2}\right)\left|x_{s}\right| .
$$

On multiplying the above inequality with Inequality (3), then

$$
\left|\left(A_{0}^{2}\right)_{t t}-\bar{a}^{2}\right|\left|\left(A_{0}^{2}\right)_{s s}-\bar{a}^{2}\right|\left|x_{t}\right|\left|x_{s}\right| \leq r_{t}\left(A_{0}^{2}\right) r_{s}\left(A_{0}^{2}\right)\left|x_{t}\right|\left|x_{s}\right| .
$$

Note that $\left|x_{t}\right|\left|x_{s}\right|>0$, then

$$
\left|\left(A_{0}^{2}\right)_{t t}-\bar{a}^{2}\right|\left|\left(A_{0}^{2}\right)_{s S}-\bar{a}^{2}\right| \leq r_{t}\left(A_{0}^{2}\right) r_{s}\left(A_{0}^{2}\right),
$$

which contradicts Inequality (1). Therefore, $A$ is non-singular.

From Lemma 1, we can obtain a new eigenvalue inclusion set for matrices with a c.m.d.

Theorem 4. Let $A=\left[a_{i j}\right] \in \mathbb{C}^{n \times n}$ with $a_{i i}=\bar{a}$ for all $i \in N$, and $n \geq 2$. Then,

$$
\sigma(A) \subseteq \bar{\Omega}(A)=\bigcup_{i, j \in N, i \neq j} \bar{\Omega}_{i, j}(A),
$$

where

$$
\begin{gathered}
\bar{\Omega}_{i, j}(A)=\left\{z \in \mathbb{C}:\left|z-\bar{a}-\sqrt{\left(A_{0}^{2}\right)_{i i}}\right|\left|z-\bar{a}+\sqrt{\left(A_{0}^{2}\right)_{i i}}\right|\left|z-\bar{a}-\sqrt{\left(A_{0}^{2}\right)_{j j}}\right|\right. \\
\left.\left|z-\bar{a}+\sqrt{\left(A_{0}^{2}\right)_{j j}}\right| \leq r_{i}\left(A_{0}^{2}\right) r_{j}\left(A_{0}^{2}\right)\right\},
\end{gathered}
$$

and $A_{0}=A-\bar{a} I$.

Proof. Suppose that $\lambda \in \sigma(A)$, then $\lambda I-A$ is singular. If $\lambda \notin \bar{\Omega}(A)$, then $\lambda \notin \bar{\Omega}_{i j}(A)$ for any $i, j \in N, i \neq j$, which leads to that for any $i, j \in N, i \neq j$,

$$
\left|z-\bar{a}-\sqrt{\left(A_{0}^{2}\right)_{i i}}\right|\left|z-\bar{a}+\sqrt{\left(A_{0}^{2}\right)_{i i}}\right|\left|z-\bar{a}-\sqrt{\left(A_{0}^{2}\right)_{j j}}\right|\left|z-\bar{a}+\sqrt{\left(A_{0}^{2}\right)_{j j}}\right|>r_{i}\left(A_{0}^{2}\right) r_{j}\left(A_{0}^{2}\right),
$$

that is,

$$
\left|(z-\bar{a})^{2}-\left(A_{0}^{2}\right)_{i i}\right|\left|(z-\bar{a})^{2}-\left(A_{0}^{2}\right)_{j j}\right|>r_{i}\left(A_{0}^{2}\right) r_{j}\left(A_{0}^{2}\right) .
$$

From Lemma 1 , we have that $\lambda I-A$ is non-singular. This contradicts that $\lambda I-A$ is singular. Hence, $\lambda \in \bar{\Omega}(A)$.

We now give a comparison between the new eigenvalue set $\bar{\Omega}(A)$ and the set $\Omega(A)$ in Theorem 1 .

Theorem 5. Let $A=\left[a_{i j}\right] \in \mathbb{C}^{n \times n}$ with $a_{i i}=\bar{a}$ for any $i \in N$, and $n \geq 2$. Then,

$$
\bar{\Omega}(A) \subseteq \Omega(A) .
$$


Proof. Suppose that $z \in \bar{\Omega}(A)$, then there exist $i, j \in N$ with $i \neq j$ and $z \in \bar{\Omega}_{i j}(A)$, that is,

$$
\begin{gathered}
\left|z-\bar{a}-\sqrt{\left(A_{0}^{2}\right)_{i i}}\right|\left|z-\bar{a}+\sqrt{\left(A_{0}^{2}\right)_{i i}}\right|\left|z-\bar{a}-\sqrt{\left(A_{0}^{2}\right)_{j j}}\right| \\
\left|z-\bar{a}+\sqrt{\left(A_{0}^{2}\right)_{j j}}\right| \leq r_{i}\left(A_{0}^{2}\right) r_{j}\left(A_{0}^{2}\right) .
\end{gathered}
$$

Equivalently,

$$
\left|(z-\bar{a})^{2}-\left(A_{0}^{2}\right)_{i i}\right|\left|(z-\bar{a})^{2}-\left(A_{0}^{2}\right)_{j j}\right| \leq r_{i}\left(A_{0}^{2}\right) r_{j}\left(A_{0}^{2}\right) .
$$

If $r_{i}\left(A_{0}^{2}\right) r_{j}\left(A_{0}^{2}\right)=0$, then $(z-\bar{a})^{2}=\left(A_{0}^{2}\right)_{i i}$ or $(z-\bar{a})^{2}=\left(A_{0}^{2}\right)_{j j}$. We can get $z \in \Omega_{i}(A)$ or $z \in \Omega_{j}(A)$ and hence $z \in \Omega_{i}(A) \cup \Omega_{j}(A)$. If $r_{i}\left(A_{0}^{2}\right) r_{j}\left(A_{0}^{2}\right)>0$, we have from Inequality (8),

$$
\left(\frac{\left|(z-\bar{a})^{2}-\left(A_{0}^{2}\right)_{i i}\right|}{r_{i}\left(A_{0}^{2}\right)}\right)\left(\frac{\left|(z-\bar{a})^{2}-\left(A_{0}^{2}\right)_{j j}\right|}{r_{j}\left(A_{0}^{2}\right)}\right) \leq 1,
$$

that is, $\left|(z-\bar{a})^{2}-\left(A_{0}^{2}\right)_{i i}\right| \leq r_{i}\left(A_{0}^{2}\right)$ or $\left|(z-\bar{a})^{2}-\left(A_{0}^{2}\right)_{j j}\right| \leq r_{j}\left(A_{0}^{2}\right)$. Hence, $z \in \Omega_{i}(A)$ or $z \in \Omega_{j}(A)$, consequently, $z \in \Omega_{i}(A) \cup \Omega_{j}(A)$ and

$$
\bar{\Omega}_{i j}(A) \subseteq \Omega_{i}(A) \bigcup \Omega_{j}(A) .
$$

As Equation (9) holds for any $i$ and $j(i \neq j)$ in $N$, therefore $\bar{\Omega}(A) \subseteq \Omega(A)$.

Example 1. Consider the matrix $A$ (the matrix $A_{4}$ in [9]),

$$
A=\left[\begin{array}{cccc}
2 & i & -3 & -i \\
0 & 2 & 1 & -5 i \\
4 & 1 & 2 & 2 \\
i & -1 & 1 & 2
\end{array}\right]
$$

the sets $\Gamma(A), \mathcal{K}(A), \Omega(A)$, and $\bar{\Omega}(A)$ are shown in Figure 1, where $\Gamma(A)$ is represented by the outside boundary, $\mathcal{K}(A)$ by the middle, $\Omega(A)$ by the inner, and $\bar{\Omega}(A)$ is filled. The exact eigenvalues are plotted with asterisks. It is easy to see that

$$
\bar{\Omega}(A) \subset \Omega(A) \subset \mathcal{K}(A) \subset \Gamma(A) .
$$

This example shows that the the new eigenvalue inclusion set in Theorem 4 is tighter than the Geršgorin set $\Gamma(A)$, the Brauer set $\mathcal{K}(A)$ and the set $\Omega(A)$ obtained in [9].

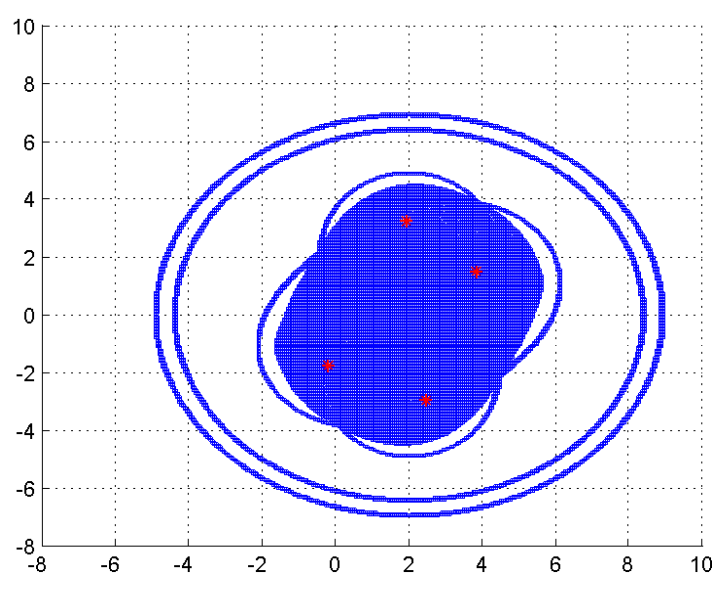

Figure 1. $\bar{\Omega}(A) \subset \Omega(A) \subset \mathcal{K}(A) \subset \Gamma(A)$. 
Remark 1. From Theorems 3 and 5, we have that

$$
\bar{\Omega}(A) \subseteq \Omega(A), \bar{\Omega}\left(A^{T}\right) \subseteq \Omega\left(A^{T}\right),\left(\bar{\Omega}(A) \bigcap \bar{\Omega}\left(A^{T}\right)\right) \subseteq\left(\Omega(A) \bigcap \Omega\left(A^{T}\right)\right)
$$

and

$$
\Omega^{2}(A) \subseteq \Omega^{1}(A) \subseteq\left(\Omega(A) \bigcap \Omega\left(A^{T}\right)\right) .
$$

Note here that $\Omega^{1}(A)=\Omega^{1}\left(A^{T}\right)$ and $\Omega^{2}(A)=\Omega^{2}\left(A^{T}\right)$. However, the sets $\Omega^{2}(A)$ and $\bar{\Omega}(A) \cap \bar{\Omega}\left(A^{T}\right)$ (also $\Omega^{1}(A)$ and $\bar{\Omega}(A) \cap \bar{\Omega}\left(A^{T}\right)$ ) cannot be compared with each other. In fact, we also consider the matrix $A$ in Example 1, and draw $\Omega^{2}(A)$, and $\bar{\Omega}(A) \cap \bar{\Omega}\left(A^{T}\right)$ in Figures 2 and 3. It is not difficult to see that

$$
\Omega^{2}(A) \nsubseteq \subset \bar{\Omega}(A) \bigcap \bar{\Omega}\left(A^{T}\right)
$$

and

$$
\bar{\Omega}(A) \bigcap \bar{\Omega}\left(A^{T}\right) \not \Omega^{2}(A) .
$$

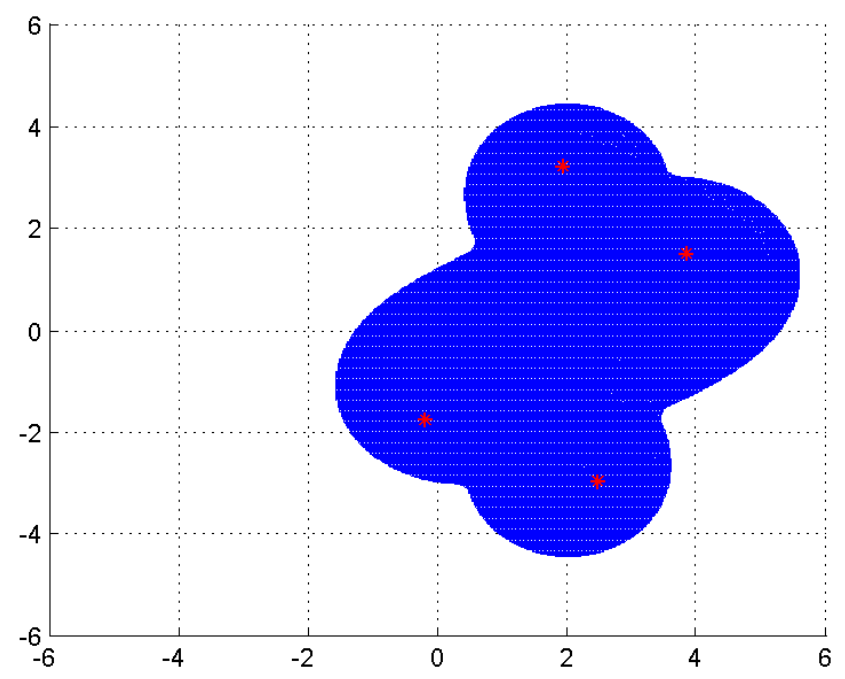

Figure 2. $\Omega^{2}(A)$.

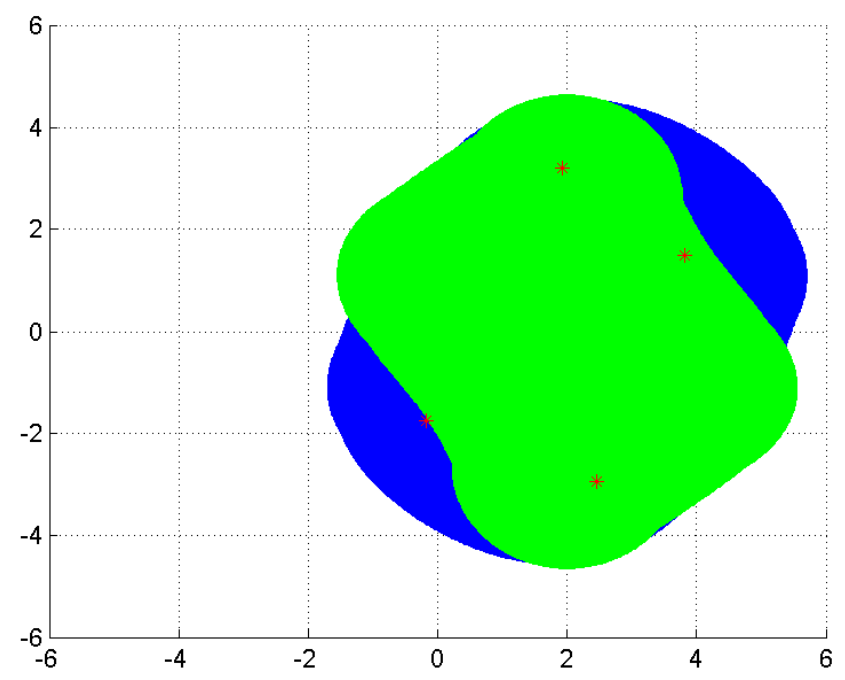

Figure 3. $\bar{\Omega}(A) \cap \bar{\Omega}\left(A^{T}\right)$. 


\section{Eigenvalue Inclusion Set for Toeplitz Matrices}

Toeplitz matrices, a subclass of matrices with a c.m.d., arise in many fields of application [12-18], such as probability and statistics, signal processing, differential and integral equations, Markov chains, Padé approximation, etc. For example, consider an assigned Lebesgue integrable function $f$ defined on the fundamental interval $I=[-\pi, \pi)$ and periodically extended to the whole real axis, and the Fourier coefficients $a_{k}$ of $f$ that is

$$
a_{k}=\frac{1}{2 \pi} \int_{-\pi}^{\pi} f(x) e^{-\mathbf{i} k x} d x,\left(\mathbf{i}^{2}=-1\right)
$$

where $k$ is an integer number. From the coefficients $a_{k}$ one can build the infinite dimensional Toeplitz matrix $\operatorname{Tn}(f)$ with entries $(\operatorname{Tn}(f))_{s t}=a_{s-t}, s, t=1,2 \ldots, n[12,13,16]$.

Toeplitz matrices are constant along all their NW-SE diagonals [7,9], i.e., a Toeplitz matrix $T \in \mathbb{C}^{n \times n}$ has the following form:

$$
T=\left[\begin{array}{ccccc}
t_{0} & t_{1} & t_{2} & \cdots & t_{n-1} \\
t_{-1} & t_{0} & t_{1} & \cdots & t_{n-2} \\
\vdots & \ddots & \ddots & \ddots & \vdots \\
t_{2-n} & \cdots & t_{-1} & t_{0} & t_{1} \\
t_{1-n} & \cdots & t_{-2} & t_{-1} & t_{0}
\end{array}\right]
$$

Indeed, if $f$ is a real valued function, we have $a_{k}=\bar{a}_{-k}$ and, consequently, $T_{n}(f)$ is Hermitian; moreover, if $f(x)=f(-x)$, then the coefficients $a_{k}$ are real and $T_{n}(f)$ is symmetric. The following result can be found in $[12,19]$ and in a multilevel setting in [16,17].

Theorem $6([17,19])$. Let $\lambda_{j}^{(n)}$ be the eigenvalues of $T_{n}(f)$ sorted in nondecreasing order, and $m_{f}=$ ess inf $f$, $M_{f}=\operatorname{ess} \sup f$.

a. If $m_{f}<M_{f}$, then $\lambda_{j}^{(n)} \in\left(m_{f}, M_{f}\right)$ for every $j$ and $n$; if $m_{f}=M_{f}$, then $f$ is constant and trivially $T_{n}(f)=m_{f} I_{n}$ with $I_{n}$ identity of size $n$;

b. The following asymptotic relationships hold: $\lim _{n \rightarrow \infty} \lambda_{1}^{(n)}=m_{f}, \lim _{n \rightarrow \infty} \lambda_{n}^{(n)}=M_{f}$.

Furthermore, there exist further results establishing precisely how fast the convergence holds $[13,17]$. Since in applications (differential and fractional operators/equations, shift-invariant integral operators/equations, signal and image processing etc.) often the underlying Toeplitz matrices have large size $n$, then the results in $[12,13,16,17]$ are difficult to beat and improved. When $f$ is complex-values the theory is more complicated and in that case the convex hull of the essential range of $f$ plays a role (see $[13,18])$. Obviously, a Toeplitz matrix is persymmetric. Here, we call $A$ persymmetric if $A$ is symmetric with respect to the main anti-diagonal [9]. Furthermore, the square of a Toeplitz matrix $T$ is not necessary Toeplitz, but it is persymmetric.

In [9], Melman applied the eigenvalue inclusion Theorem (Theorem 1) of matrices with a c.m.d. to Toeplitz matrices, and obtained the following simpler form of the eigenvalue inclusion set.

Theorem 7 ([9] Theorem 3.1). Let $T=\left[t_{i j}\right] \in \mathbb{C}^{n \times n}$ be a Toeplitz matrix and $t_{i i}=\bar{t}, n \geq 2$. Then,

$$
\sigma(T) \subseteq \Omega(T)=\bigcup_{i=1}^{\left\lceil\frac{n}{2}\right\rceil} \Omega_{i}(T),
$$

where

$$
\begin{gathered}
\Omega_{i}(T)=\left\{z \in \mathbb{C}:\left|z-\bar{t}-\sqrt{\left(T_{0}^{2}\right)_{i i}}\right|\left|z-\bar{t}+\sqrt{\left(T_{0}^{2}\right)_{j j}}\right| \leq v_{i}\left(T_{0}^{2}\right)\right\}, \\
T_{0}=T-\bar{t} I, v_{i}\left(T_{0}^{2}\right)=\max \left\{r_{i}\left(T_{0}^{2}\right), r_{n-i+1}\left(T_{0}^{2}\right)\right\},
\end{gathered}
$$


and

$$
\left\lceil\frac{n}{2}\right\rceil=\left\{\begin{array}{cc}
\frac{n}{2}, & \text { if } n \text { is even, } \\
\frac{n+1}{2}, & \text { if } n \text { is odd. }
\end{array}\right.
$$

Furthermore, $\Omega(T) \subseteq \mathcal{K}(T) \subseteq \Gamma(T)$.

Next, by applying Theorem 4 to Toeplitz matrices, we obtain a new eigenvalue inclusion set.

Theorem 8. Let $T=\left[t_{i j}\right] \in \mathbb{C}^{n \times n}$ be a Toeplitz matrix with $t_{11}=\bar{t}$ and $n \geq 2$. Then,

$$
\sigma(T) \subseteq \bar{\Omega}(T)=\left(\bigcup_{i, j \in\left\lceil\frac{n}{2}\right\rceil, i \neq j} \bar{\Omega}_{i j}^{1}(T)\right) \bigcup\left(\bigcup_{i \in\left\lceil\frac{n}{2}\right\rceil} \bar{\Omega}_{i}(T)\right),
$$

where

$$
\begin{aligned}
& \bar{\Omega}_{i j}^{1}(T)=\left\{z \in \mathbb{C}: \quad\left|z-t_{0}-\sqrt{\left(T_{0}^{2}\right)_{i i}}\right|\left|z-t_{0}+\sqrt{\left(T_{0}^{2}\right)_{i i}}\right|\right. \\
& \left.\left|z-t_{0}-\sqrt{\left(T_{0}^{2}\right)_{j j}}\right|\left|z-t_{0}+\sqrt{\left(T_{0}^{2}\right)_{j j}}\right| \leq V_{i}\left(T_{0}^{2}\right) V_{j}\left(T_{0}^{2}\right)\right\}, \\
& \bar{\Omega}_{i}(T)=\left\{z \in \mathbb{C}:\left(\left|z-t_{0}-\sqrt{\left(T_{0}^{2}\right)_{i i}}\right|\left|z-t_{0}+\sqrt{\left(T_{0}^{2}\right)_{i i}}\right|\right)^{2} \leq r_{i}\left(T_{0}^{2}\right) r_{n-i+1}\left(T_{0}^{2}\right)\right\}, \\
& V_{i}\left(T_{0}^{2}\right)=\max \left\{r_{i}\left(T_{0}^{2}\right), r_{n-i+1}\left(T_{0}^{2}\right)\right\}, \text { and } T_{0}=T-t_{0} I .
\end{aligned}
$$

Proof. Since $T$ is Toeplitz and $T_{0}=T-\bar{t} I$, we have that $T_{0}$ is also Toeplitz and $T_{0}^{2}$ is persymmetric. Therefore, the main diagonal of $T_{0}^{2}$ has at most $\left\lceil\frac{n}{2}\right\rceil$ distinct values, and $\left(T_{0}^{2}\right)_{i i}=\left(T_{0}^{2}\right)_{n-i+1, n-i+1}$ for $i=1,2, \ldots,\left\lceil\frac{n}{2}\right\rceil$. Hence, by Theorem 4 and Equation (6), for any $\lambda \in \sigma(T), \lambda \in \bar{\Omega}(T)=\bigcup_{i, j \in N, i \neq j} \bar{\Omega}_{i j}(T)$. For the case $i, j \in\left\{1,2, \ldots,\left\lceil\frac{n}{2}\right\rceil\right\}, j \neq i$, we have

$$
\left|(\lambda-\bar{t})^{2}-\left(T_{0}^{2}\right)_{i i}\right|\left|(\lambda-\bar{t})^{2}-\left(T_{0}^{2}\right)_{j j}\right| \leq r_{i}\left(T_{0}^{2}\right) r_{j}\left(T_{0}^{2}\right)
$$

For the case $i \in\left\{1,2, \ldots,\left\lceil\frac{n}{2}\right\rceil\right\}, j \in N \backslash\left\{1,2, \ldots,\left\lceil\frac{n}{2}\right\rceil\right\}, j \neq n-i+1$, we have

$$
\left|(\lambda-\bar{t})^{2}-\left(T_{0}^{2}\right)_{i i}\right|\left|(\lambda-\bar{t})^{2}-\left(T_{0}^{2}\right)_{n-j+1, n-j+1}\right| \leq r_{i}\left(T_{0}^{2}\right) r_{n-j+1}\left(T_{0}^{2}\right) .
$$

Note that $\left(T_{0}^{2}\right)_{j j}=\left(T_{0}^{2}\right)_{n-j+1, n-j+1}$, then

$$
\left|(\lambda-\bar{t})^{2}-\left(T_{0}^{2}\right)_{i i}\right|\left|(\lambda-\bar{t})^{2}-\left(T_{0}^{2}\right)_{j j}\right| \leq r_{i}\left(T_{0}^{2}\right) r_{n-j+1}\left(T_{0}^{2}\right) .
$$

From Inequalities (10) and (11), we can get that

$$
\left|(\lambda-\bar{t})^{2}-\left(T_{0}^{2}\right)_{i i}\right|\left|(\lambda-\bar{t})^{2}-\left(T_{0}^{2}\right)_{j j}\right| \leq r_{i}\left(T_{0}^{2}\right) V_{j}\left(T_{0}^{2}\right),
$$

where $V_{j}\left(T_{0}^{2}\right)=\max \left\{r_{j}\left(T_{0}^{2}\right), r_{n-j+1}\left(T_{0}^{2}\right)\right\}$. Similarly, we obtain

$$
\left|(\lambda-\bar{t})^{2}-\left(T_{0}^{2}\right)_{n-i+1, n-i+1}\right|\left|(\lambda-\bar{t})^{2}-\left(T_{0}^{2}\right)_{j j}\right| \leq r_{n-i+1, n-i+1}\left(T_{0}^{2}\right) V_{j}\left(T_{0}^{2}\right) .
$$

From $\left(T_{0}^{2}\right)_{i i}=\left(T_{0}^{2}\right)_{n-i+1, n-i+1}$, Inequalities (12) and (13), we could easily get, for any $i, j \in\left\{1,2, \ldots,\left\lceil\frac{n}{2}\right\rceil\right\}$ and $j \neq i$,

$$
\left|(\lambda-\bar{t})^{2}-\left(T_{0}^{2}\right)_{i i}\right|\left|(\lambda-\bar{t})^{2}-\left(T_{0}^{2}\right)_{j j}\right| \leq V_{i}\left(T_{0}^{2}\right) V_{j}\left(T_{0}^{2}\right) .
$$


Furthermore, for any $i \in\left\{1,2, \ldots,\left\lceil\frac{n}{2}\right\rceil\right\}, j=n-i+1$,

$$
\left|(\lambda-\bar{t})^{2}-\left(T_{0}^{2}\right)_{i i}\right|\left|(\lambda-\bar{t})^{2}-\left(T_{0}^{2}\right)_{n-i+1, n-i+1}\right| \leq r_{i}\left(T_{0}^{2}\right) r_{n-i+1}\left(T_{0}^{2}\right)
$$

which is equivalent to

$$
\left|(\lambda-\bar{t})^{2}-\left(T_{0}^{2}\right)_{i i}\right|\left|(\lambda-\bar{t})^{2}-\left(T_{0}^{2}\right)_{i, i}\right| \leq r_{i}\left(T_{0}^{2}\right) r_{n-i+1}\left(T_{0}^{2}\right),
$$

that is,

$$
\left(\left|z-t_{0}-\sqrt{\left(T_{0}^{2}\right)_{i i}}\right|\left|z-t_{0}+\sqrt{\left(T_{0}^{2}\right)_{i i}}\right|\right)^{2} \leq r_{i}\left(T_{0}^{2}\right) r_{n-i+1}\left(T_{0}^{2}\right) .
$$

The conclusion follows from Inequalities (14) and (15).

From Theorems 5, 7 and 8, we can obtain easily the comparison results as follows.

Theorem 9. Let $T=\left[t_{i j}\right] \in \mathbb{C}^{n \times n}$ be a Toeplitz matrix with $t_{11}=\bar{t}$ and $n \geq 2$. Then,

$$
\bar{\Omega}(T) \subseteq \Omega(T) \subseteq \mathcal{K}(T) \subseteq \Gamma(T) .
$$

Example 2. Consider the Toeplitz matrix $Q$ in [9]:

$$
Q=\left[\begin{array}{cccc}
6 & 1 & -1 & -2 i \\
0 & 6 & 1 & -1 \\
-1 & 0 & 6 & 1 \\
4 & -1 & 0 & 6
\end{array}\right]
$$

In Figure 4, the sets $\Gamma(Q), \mathcal{K}(Q), \Omega(Q)$, and $\bar{\Omega}(Q)$ are shown, where $\Gamma(Q)$ is represented by the outside boundary, $\mathcal{K}(Q)$ by the middle, $\Omega(Q)$ by the inner, and $\bar{\Omega}(Q)$ is filled. The exact eigenvalues are plotted with asterisks. As we can see,

$$
\bar{\Omega}(Q) \subset \Omega(Q) \subset \mathcal{K}(Q) \subset \Gamma(Q) .
$$

This example shows that the new eigenvalue inclusion set in Theorem 8 is tighter than the set obtained in [9], the Geršgorin set and the Brauer set for a Toeplitz matrix.

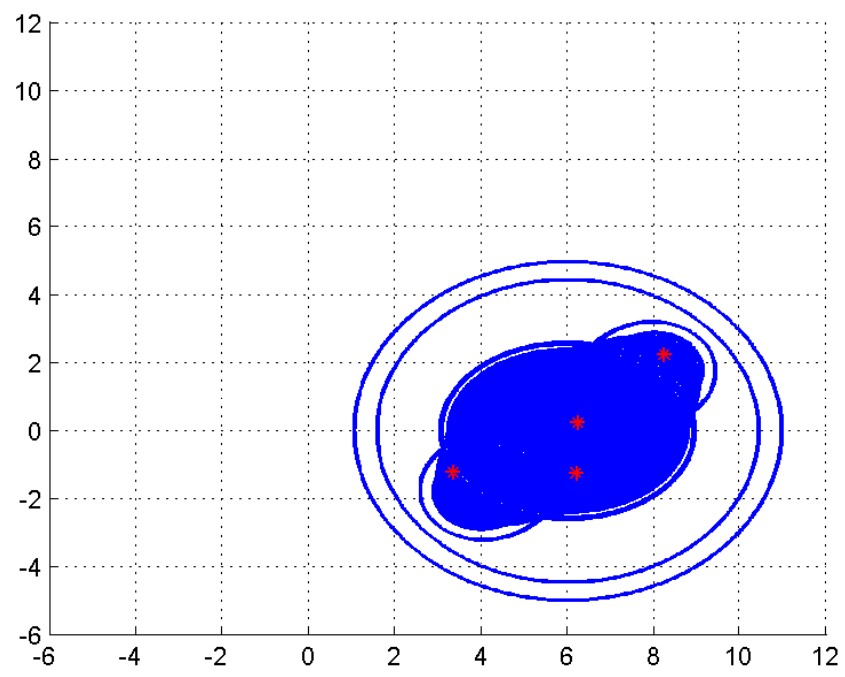

Figure 4. $\bar{\Omega}(Q) \subset \Omega(Q) \subset \mathcal{K}(Q) \subset \Gamma(Q)$. 


\section{Conclusions}

In this paper, we obtain a new eigenvalue inclusion set for matrices with a c.m.d. We then apply this result to Toeplitz matrices, and get a set including all eigenvalues of Toeplitz matrices. Although they needs more computations to obtain the new eigenvalue sets than those in [9], the new sets capture all eigenvalues more precisely than those in [9].

Author Contributions: All the authors inferred the main conclusions and approved the current version of this manuscript.

Funding: This research was funded by the Applied Basic Research Programs of Science and Technology Department of Yunnan Province (grant number 2018FB001), Outstanding Youth Cultivation Project for Yunnan Province (grant number 2018YDJQ021), Program for Excellent Young Talents in Yunnan University, and Yunnan Provincial Ten Thousands Plan Young Top Talents.

Acknowledgments: We would like to thank the editor Kathy Wang, and the referees for their detailed and helpful comments.

Conflicts of Interest: The authors declare no conflict of interest.

\section{References}

1. Brauer, A. Limits for the characteristic roots of a matrix II. Duke Math. J. 1947, 14, 21-26. [CrossRef]

2. Cvetković, L. H-matrix theory vs. eigenvalue localization. Numer. Algorithms 2006, 42, 229-245. [CrossRef]

3. Cvetković, L.; Kostić, V.; Bru, R.; Pedroche, F. A simple generalization of Geršgorin's theorem. Adv. Comput. Math. 2011, 35, 271-280. [CrossRef]

4. Cvetković, L.; Kostić, V.; Varga, R.S. A new Geršgorin-type eigenvalue inclusion set. Electron. Trans. Numer. Anal. 2004, 18, 73-80.

5. Geršgorin, S. Über die Abgrenzung der Eigenwerte einer Matrix. Izv. Akad. Nauk SSSR Ser. Mat. 1931, 1,749-754.

6. Li, C.Q.; Li, Y.T. Generalizations of Brauer's eigenvalue localization theorem. Electron. J. Linear Algebra 2011, 22, 1168-1178. [CrossRef]

7. Li, C.Q.; Li, Y.T. New regions including eigenvalues of Toeplitz matrices. Linear Multilinear Algebra 2014, 62, 229-241. [CrossRef]

8. Melman, A. Modified Gershgorin Disks for Companion Matrices. Siam Rev. 2012, 54, 355-373.

9. Melman, A. Ovals of Cassini for Toeplitz matrices. Linear Multilinear Algebra 2012, 60, 189-199. [CrossRef]

10. Sang, C.L.; Zhao, J.X. Eventually DSDD Matrices and Eigenvalue Localization. Symmetry 2018, 10, 448. [CrossRef]

11. Varga, R.S. Geř̌gorin and His Circles; Springer: Berlin, Germany, 2004.

12. Di Benedetto, F.; Fiorentino, G.; Serra, S. CG preconditioning for Toeplitz matrices. Comput. Math. Appl. 1993, 25, 35-45. [CrossRef]

13. Böttcher, A.; Grudsky, S. On the condition numbers of large semi-definite Toeplitz matrices. Linear Algebra Appl. 1998, 279, 285-301. [CrossRef]

14. Bunch, J.R. Stability of methods for solving Toeplitz systems of equations. SIAM J. Sci. Stat. Comput. 1985, 6,349-364. [CrossRef]

15. Pourahmadi, M. Remarks on extreme eigenvalues of Toeplitz matrices. Int. J. Math. Math. Sci. 1988, 11, 23-26. [CrossRef]

16. Serra, S. Preconditioning strategies for asymptotically ill-conditioned block Toeplitz systems. BIT 1994, 34, 579-594. [CrossRef]

17. Serra, S. On the Extreme Eigenvalues of Hermitian (Block) Toeplitz Matrices. Linear Algebra Appl. 1998, 270, 109-129. [CrossRef]

18. Capizzano, S.S.; Tilli, P. Extreme singular values and eigenvalues of non-Hermitian block Toeplitz matrices. J. Comput. Appl. Math. 1999, 108, 113-130. [CrossRef]

19. Grenander, U.; Szegö, G. Toeplitz Forms and Their Applications, 2nd ed.; Chelsea: New York, NY, USA, 1984.

(C) 2018 by the authors. Licensee MDPI, Basel, Switzerland. This article is an open access article distributed under the terms and conditions of the Creative Commons Attribution (CC BY) license (http://creativecommons.org/licenses/by/4.0/). 